\title{
Dairy cow handling facilities and the perception of Beef Quality Assurance on Colorado dairies
}

\author{
A. E. Adams, ${ }^{*}$ F. J. Olea-Popelka,† T. Grandin, ${ }^{*}$ D. R. Woerner, ${ }^{*}$ and I. N. Roman-Muniz ${ }^{* 1}$ \\ *Department of Animal Sciences, College of Agricultural Sciences, and \\ †Department of Clinical Sciences, College of Veterinary Medicine and Biomedical Sciences, Colorado State University, Fort Collins 80523
}

\begin{abstract}
A survey was conducted on Colorado dairies to assess attitudes and practices regarding Dairy Beef Quality Assurance (DBQA). The objectives were to (1) assess the need for a new handling facility that would allow all injections to be administered via DBQA standards; (2) establish if Colorado dairy producers are concerned with DBQA; and (3) assess differences in responses between dairy owners and herdsmen. Of the 95 dairies contacted, 20 (21\%) agreed to participate, with a median herd size of 1,178 . When asked to rank the following 7 traits - efficiency, animal safety, human safety, ease of animal handling, ease of operation, inject per Beef Quality Assurance (BQA) procedures, and cost - in order of priority when designing a new handling facility, human and animal safety were ranked highest in priority (first or second) by the majority of participants, with ease of animal handling and efficiency ranked next. Interestingly, the administration of injections per BQA standards was ranked sixth or seventh by most participants. Respondents estimated the average annual income from the sale of cull cows to be $4.6 \%$ of all dairy income, with $50 \%$ receiving at least one carcass discount or condemnation in the past 12 mo. Although almost all of the participating dairy farmers stated that the preferred injection site for medications was the neck region, a significant number admitted to using alternate injection sites. In contrast, no difference was found between responses regarding the preferred and actual location for intravenous injections. Although most participating producers are aware of BQA injection guidelines, they perceive efficiency as more important, which could result in injections being administered in locations not promoted by BQA. Dairy owners and herdsmen disagreed in whether or not workers had been injured in the animal handling area in the last $12 \mathrm{mo}$. Handling facilities that allow for an efficient and safe way to administer drugs according
\end{abstract}

Received June 14, 2013.

Accepted October 30, 2013

${ }^{1}$ Corresponding author: inromanm@colostate.edu to BQA guidelines and educational opportunities that highlight the effect of improved DBQA on profitability could prove useful. Dairy producers play a key role in ensuring that dairy beef is safe and high quality, and just as they are committed to producing safe and nutritious milk for their customers, they should be committed to producing the best quality beef.

Key words: dairy beef quality assurance, dairy cow, dairy handling facility

\section{INTRODUCTION}

Dairy cows are handled more regularly than most other livestock animals, due in large part to the daily milking routine, routine veterinary exams, and reproductive management (Lindahl et al., 2013). Excluding the milking routine, the frequency of handling likely depends on the practices on each individual dairy. For example, herds employing intensive reproductive programs such as timed AI and estrous synchronization often handle their cattle on a daily basis; in contrast, cows on other dairies may only be handled weekly during routine veterinary exams. Regardless of the reason for handling, a working facility that meets the needs of a particular dairy is necessary. The best working facility for a particular dairy depends on herd size, expected treatment regimen, housing layout, labor force, and the frequency of handling (Bickert et al., 2000). The most common facilities found on dairy operations are singlefile chutes, fence-line stanchions (headlocks), and herringbone palpation rails (management rails) (Bickert et al., 2000). Management rails are relatively simple and inexpensive to design and consist of a breast-rail and a rump-rail running parallel to each other with the front and back gates angled, similar to a herringbone milking parlor, for ease of loading (Wagner-Storch and Palmer, 2002). Appropriate handling facilities not only expedite procedures, make the dairy more efficient, and improve dairy welfare, but could also improve dairy beef quality and safety by allowing treatments to be administered in a manner that adheres to Beef Quality Assurance (BQA) principles.

The BQA program is designed to ensure that dairy and beef cattle are produced in a manner that results 
in safe, high-quality, and consistent beef products for consumers (Glaze and Chahine, 2009). The BQA program provides guidelines for producers to follow (BQA, 2012). Even though most producers are aware that the ideal location to administer injections for BQA is the neck, they continue to give them in the hind quarter due to ease of administration and management (Tozer et al., 2005). Such practices greatly compromise dairy BQA (DBQA) and the image of the dairy industry. The National Market Cow and Bull Beef Quality Audit listed injection-site lesion prevalence as a leading BQA challenge in 2007 (NCBA, 2007), with $11 \%$ of dairy cows having visible injection-site lesions. Similarly, Roeber (2003) reported that injection-site lesions rank second in importance of leading BQA issues, with only the occurrence of antimicrobial residues being of greater concern. Results from the National Beef Quality Audit (NBQA, 2011), a survey completed by beef and dairy producers, indicate that the preferred location for all injections is the neck region $(87.0 \%)$, with only $4.9 \%$ of producers preferring the top of the hip and $1.7 \%$ preferring the lower rear leg. However, when considering only the answers submitted by dairy producers, $46.4 \%$ of respondents identified the neck region as the preferred location for injections, with 18.6 and $22.1 \%$ preferring the top of the hip and the lower rear leg, respectively. Moreover, research has shown that dairy cow carcasses have significantly more injection-site lesions in outside round muscles than beef cow carcasses (49 and 26\%, respectively), illustrating that BQA practices are lacking on many dairy operations (Roeber et al., 2002).

Although the majority of income on a dairy farm is generated from the sale of liquid milk, other sources of income include the sale of cull cows and bulls for slaughter. It has been reported that cull cows and bulls comprise $4 \%$ of the income of dairy sales (Ahola et al., 2011). With an average replacement rate of 30 to $35 \%$, cull dairy cows accounted for $10.9 \%$ of the beef animals slaughtered in early 2013 (USDA, 2013). A survey examining California dairy operations found that producers were hesitant to participate in a quality assurance program, primarily for financial reasons, indicating that the costs were likely to outweigh any benefits from the program (Payne et al., 1999). This logic is in direct disagreement with projections that indicate that injection-site lesions result in an astounding $\$ 9,000,000$ loss to the beef and dairy industries (Roeber et al., 2002). It has been estimated that quality defects were responsible for a deduction of $\$ 68.82$ per cow, with injection site blemishes accounting for $\$ 11.49$ to $\$ 13.82$ of that loss (Roeber et al., 2001; Tozer et al., 2005; Glaze and Chahine, 2009; Ahola et al., 2011). These findings support the argument that dairy producers would benefit financially if they were to implement and consistently follow a DBQA program on their operations.

In the past 6 decades, dairy production in the United States has become more efficient and has experienced consolidation, with total cow and farm numbers decreasing while herds increase in size (von Keyserlingk et al., 2013). This consolidation trend has contributed to increased reliance on hired labor for managing the dairy and, in many instances, has created a disconnection between the owners of the dairy and those who handle the animals. The distance between ownership and the employees handling cows could result in different perspectives regarding the efficiency and safety of working facilities.

The primary objectives of the present study were (1) to assess the need for a new type of handling facility for working dairy cows, similar to that of a management rail, that would allow all injections to be administered via DBQA standards; (2) to establish whether Colorado dairy producers were concerned with DBQA; and (3) to assess the differences in responses by dairy owners and management compared with herd personnel. A secondary aim of the study was to describe the current trends in management practices, including housing systems, culling decisions, estrous synchronization programs, and cull-cow marketing options, on Colorado dairy operations.

\section{MATERIALS AND METHODS}

A survey was developed to assess the potential need for a new handling facility that would allow for dairy operations to more easily follow DBQA standards. Survey questions were developed to help researchers assess the current needs and uses of working facilities on Colorado dairies, to help describe current management and housing practices that could affect dairy beef quality, and to determine the importance of DBQA on dairy operations. To obtain basic information about the Colorado dairies surveyed, questions were included pertaining to herd size, housing method, average stocking percentage, and general management practices. Also, several questions were included to characterize culling behavior on Colorado dairies. As one of the objectives of this study was to assess the need for a new handling facility similar to that of a management rail, producers were asked if they would be willing to install a management rail when considering building a new handling facility. The final survey consisted of 45 open- and closed-ended questions, along with some individual questions that required multiple responses. Ninetyfive dairy operations were solicited to participate in the survey by e-mail and phone, with 20 agreeing to 
participate, resulting in a response rate of $21 \%$. The survey protocol was approved by the Colorado State University Institutional Review Board Human Subjects Research Policy (\#11-2698H).

Upon approval from the dairy owner, a member of the research team visited the dairy operation and completed the questionnaire with the owner $(\mathrm{n}=20)$ and, when applicable, completed a second part of the questionnaire with the herdsman $(\mathrm{n}=15)$. The second part of the questionnaire was not completed in instances where the owner reported to be the acting herdsman. The questions for the herdsman paralleled those of the owner to detect discrepancies between upper management (owners) and those working firsthand with the animals (herdsmen). For purposes of consistency, the same researcher conducted all surveys, recording in writing the participants' responses. After visiting the first 4 dairy operations, the research team decided that a question on the actual location of intramuscular (i.m.), subcutaneous (s.c.), and intravenous (i.v.) injections should be included in the survey as a way to compare preferred (the location where the participant would like injections administered) and actual practices. Results reflect the discrepancy between the numbers of dairies visited and total responses for this question.

All data were entered into a spreadsheet and analyzed using SAS (version 9.3; SAS Institute Inc., Cary, NC). Data were categorized as either closed-ended (yes or no) or open-ended questions. Open-ended questions were designed to describe management practices (e.g., reproductive programs), and answers provided by participants were reported descriptively by frequency. All questions were organized as stated above, with the exception of continuous data (e.g., herd size) and the ranking of 7 traits of a new handling facility in order of importance. PROC UNIVARIATE in SAS was used to calculate the median herd size, along with frequencies concerning types of handling and housing facilities, and the different management practices employed on the operations. Fisher's exact test was obtained using PROC FREQ to evaluate the preferred versus actual injection site locations, as well as associations between owner and herdsman responses.

\section{RESULTS AND DISCUSSION}

The response rate of $21 \%$ in the current survey was similar to that of Buttars et al. (2006), who had a response rate of 13 to $29 \%$ when they surveyed dairy producers about biosecurity practices. However, this response rate was lower than that of Glaze and Chahine (2009), who had a response rate of $36.9 \%$ for dairy producers in Idaho who were asked about BQA principles and practices, and also lower than that of Rogers et al.
(2004) with a response rate of $31 \%$ for dairy producers surveyed on management decisions to improve quality of market cows. The dairies surveyed represent 38,210 cows, or $33.2 \%$ of the total number of cows in Colorado, according to the USDA-APHIS (2008).

Overall, the mean number of cows per herd was 1,911, with a median of 1,178 and a range of 200 to 5,500 head. The median herd size for dairies that participated in this study was slightly higher than that reported by USDA-APHIS (2008) for Colorado dairies in 2007. As the letter soliciting participants indicated that the goal of the survey was to assess the current use and needs of handling systems, it is possible that smaller dairies did not respond due to the lack of any substantial handling facility, thus resulting in smaller dairies being underrepresented in the current study. Whether or not a herd was closed (no new animals added in the previous year) or open was split approximately evenly. The majority $(\mathrm{n}=18)$ of dairies surveyed were conventional and stated routinely using antimicrobials on their operation, whereas 2 were USDA-certified organic (USDA-AMS, 2012). The estrous synchronization program most often used was double-Ovsynch $(\mathrm{n}=9)$, which was followed by Presynch-Ovsynch $(\mathrm{n}=5)$. Most dairies $(\mathrm{n}=17)$ did not used recombinant bST (rbST) for increased milk production. The percentage of dairy farms using rbST in Colorado (15\%) agrees with the national average of $15.2 \%$ reported by USDA-APHIS (2008). The use of estrous synchronization programs and rbST results in dairy cows receiving additional injections. Additional injections and the required additional animal handling could add more pressure to move animals more quickly and increase the number of injections administered in a location that is easiest for the handler to reach but that does not comply with BQA guidelines (e.g., the rear of the cow).

Of the housing options available, 17 reported housing at least some of their cows in open lots, $12 \mathrm{had}$ freestall barns with exercise lots attached, 3 used some freestall barns with no access to an open lot, and 1 reported using compost barns to house some of their herd. For those who stated using open lots to house cows, 13 provided a windbreak for their cows and 10 had a shade structure. When presented with a multiple choice question pertaining to pen stocking percentage, determined by the number of cows and the number of available headlocks, 10 respondents reported a rate of 75 to $100 \%, 9$ stated their pens were stocked between 100 and $125 \%$, and only 1 claimed a stocking percentage of 50 to $75 \%$.

When questioned about the types of handling facilities used on their dairy, 19 dairy owners said that they use headlocks, 18 reported using a chute, 10 specified using palpation rails, and 1 stated using a head-gate in 
an alley, with many operations having multiple types of handling facilities. As expected, the most common handling facility used was headlocks, which coincides with previous findings by Wagner-Storch and Palmer (2002). As the stocking rate ideal for effectively working cattle in headlocks allows enough room for every cow in a group to lock (Bickert et al., 2000), the producers reporting an average stocking percentage above $100 \%$ were not allowing for the most efficient handling system when working their cows in headlocks. Cows not locked due to the lack of an available space or those that habitually avoid being locked by eating in broken stanchions or toward the end of the pen where no locks exist will have to be restrained in another manner. In some instances, these cows will be moved out of the pen and worked through a separate facility, or will be pinned between other animals. Nine participants indicated that they would not consider installing a management rail, whereas 11 stated that they would. Producers who would not consider building a management rail indicated the need to sort cows as the main reason against building it, whereas those in favor felt that rails were safer for the humans involved and more efficient than other handling systems. The fact that the need to sort cows was a reason given by those who were not interested in installing a management rail was not surprising, as Wagner-Storch and Palmer (2002) had previously stated that producers preferred headlocks for that exact reason.

Producers were asked to rank 7 traits in order of importance $(1=$ important, $7=$ not important $)$ when designing a new handling facility (Figure 1). The majority of the producers $(n=18)$ ranked human safety as first or second in priority, and 11 ranked animal safety as first or second, with ease of animal handling and efficiency being ranked next. Interestingly, administration of injections per BQA standards was ranked sixth or seventh by most of the participants $(\mathrm{n}=15)$. With the majority of producers ranking human safety as first or second in importance when designing a new handling facility, one would have expected an increased willingness to install a management rail, given its effect on human safety. This finding may indicate that bettertargeted extension efforts are required (e.g., improving producer education on the positive effects of management rails).

The majority of owners $(\mathrm{n}=12)$ reported low milk production as the primary reason for marketing cull cows, with the remaining 8 stating reproductive problems as the primary reason. In addition, mastitis and lameness were listed as common reasons for sending cull cows to market. These results are similar to previous studies investigating culling behavior on dairies. Studies conducted by Rogers et al. (2004) in New Mexico and by Glaze and Chahine (2009) in Idaho identified low milk production and reproductive problems as the top 2 reasons for culling dairy cows.

When asked about marketing cull cows, 8 producers stated that they send all cull cows to the sale barn (auction market), whereas the other 12 reported utilizing both the auction market as well as direct sale to the slaughter plant, which was similar to culling practices reported in previous studies (Rogers et al., 2004; Glaze and Chahine, 2009). The consensus for those utilizing both avenues was that weaker cows were sent directly to the packer and all other cows went directly to the auction. Caution should be taken when sending weak or sick animals to slaughter as they are at increased risk of becoming nonambulatory during transport. Nonambulatory cattle represent a major DBQA concern, as they are no longer fit for slaughter and human consumption, as well as a welfare concern. Half of the owners surveyed reported receiving at least one carcass discount or condemnation in the past $12 \mathrm{mo}$, with the leading reason for condemnation at the packing plant being cancer. The average age of cull cows for Colorado dairies surveyed was 4.28 yr. On average, the estimated annual income from the sale of cull cows was $4.6 \%$ of total dairy income.

Both owners and herdsmen were asked about injectable medication administration practices to assess DBQA practices. As the first 4 questionnaires completed did not contain questions pertaining to the actual location of injections, not all participants provided a response to the actual location of injections. Additionally, 1 organic dairy did not provide answers for any questions pertaining to injections, stating that no injections were administered on their operation. For statistical

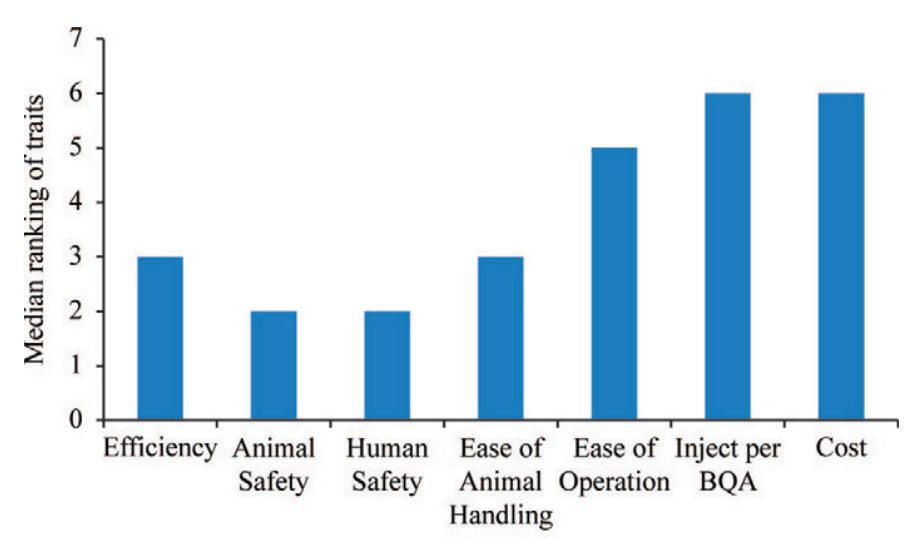

Traits that owners were asked to rank

Figure 1. Median responses of dairy owners $(n=20)$ when ranking traits of a new handling facility in order of importance between 1 and 7 , with 1 being most important and 7 being least important. BQA $=$ Beef Quality Assurance procedures. Color version available in the online PDF. 
Table 1. Preferred and actual location of subcutaneous (s.c.), intramuscular (i.m.), and intravenous (i.v.) injections as reported by Colorado dairy owners and herdsmen

\begin{tabular}{|c|c|c|c|c|c|c|c|c|c|c|}
\hline \multirow[b]{2}{*}{ Location } & \multicolumn{4}{|c|}{ Owner } & \multirow[b]{2}{*}{$P$-value } & \multicolumn{4}{|c|}{ Herdsman } & \multirow[b]{2}{*}{$P$-value } \\
\hline & Neck & $\begin{array}{l}\text { Hind quarter } \\
\left(\text { or milk vein }^{1}\right)\end{array}$ & Either & Total no. & & Neck & $\begin{array}{l}\text { Hind quarter } \\
\left(\text { or milk vein }^{1}\right)\end{array}$ & Either & Total no. & \\
\hline Actual s.c. & 8 & 0 & 7 & $15^{\mathrm{a}}$ & & 6 & 1 & 5 & 12 & \\
\hline Preferred i.m. & 11 & 4 & 0 & $15^{\mathrm{a}}$ & 0.02 & 8 & 4 & 0 & $12^{\mathrm{a}}$ & 0.02 \\
\hline Preferred i.v. & 13 & 1 & 1 & 15 & 1.00 & 10 & 2 & 0 & 12 & 1.00 \\
\hline Actual i.v. & 12 & 1 & 2 & 15 & & 10 & 1 & 1 & 12 & \\
\hline
\end{tabular}

${ }^{\mathrm{a}}$ A difference was found between the preferred and actual locations of injections $(P<0.05)$.

${ }^{1}$ In the case of i.v. injections, answers in this category refer to the milk vein.

analysis, responses were only compared for dairy owners $(\mathrm{n}=15)$ and herdsmen $(\mathrm{n}=12)$ who were asked both the preferred and actual location of s.c., i.m., and i.v. injections (Table 1). Participating dairy producers in Colorado overwhelmingly stated that the preferred location for administrating injectable medications was the neck region; however, a significant number reported that not all s.c. and i.m. injections were always administered in that location, with no significant difference being found between the preferred and actual location for i.v. injections. Previous studies have investigated the administration practices of medications on dairies, but this is the first that has included data for preferred versus actual administration, illustrating the difference between the ideal situation and what is actually carried out in practice. In the current study, the percentage of injections being administered, according to DBQA guidelines, in the neck region was smaller than that found in previous studies. Glaze and Chahine (2009) conducted a study in Idaho investigating the beef quality assurance practices of dairy operations, and found that 59 to $87 \%$ of routine i.m. and s.c. injections were administered following DBQA guidelines (Glaze and Chahine, 2009). In contrast, a similar study conducted in Pennsylvania reported that $65 \%$ of injections were administered in areas other than the neck muscle, with the majority being given in the tail-head or hip, rump, or flank areas (Tozer et al., 2005). These findings support the idea that, although they may be aware that all i.m. and s.c. injections should be given in the neck region, most dairy producers are not concerned enough about BQA to ensure that all injections are actually given in that location. In contrast, the consistency between the preferred and actual location for the administration of i.v. injections suggests that most dairy producers are not willing to compromise animal health and ensure that all i.v. injections are administered in the jugular vein instead of the milk vein, which is potentially easier to access in some instances. That said, one producer in- dicated that it was routine to administer i.v. injections via the milk vein, stating, "I know you're not going to approve, but I let the guys do it that way because it's easier for them than to have to tie the cow's head off in order to access the jugular vein," proving that, on that particular dairy, efficiency takes precedence.

One explanation as to why most dairy producers are not concerned about DBQA is the lack of an apparent incentive for them to follow the standards laid out by the BQA program. The proportion of income derived from cull cows reported by Colorado dairies $(4.6 \%)$ is only slightly higher than the national average of $4 \%$ previously reported by Ahola et al. (2011). When accounting for such a small amount of income derived from the sale of cull cows, there is little motivation for dairy producers to put more emphasis on DBQA. When asked to rank the importance of administering injections according to BQA standards, the majority of producers $(75 \%)$ ranked it last or second to last, with one producer ranking all other traits as first in priority, and ranking BQA as not important (ranked 7 out of 7 ). One producer stated, "I am in the dairy industry and have cows to produce milk, not to produce beef." Such statements make it apparent that BQA is not a priority for some dairies in Colorado. These sentiments may be mirrored in other states as well. A study conducted by Glaze and Chahine (2009) found that dairy producers did not feel that dairy animals had a major effect on the beef industry. It is possible that dairy owners do not put an emphasis on DBQA because they do not feel that dairy cows have a large enough impact on the beef industry to invest the time and resources to follow BQA protocols. Nightingale et al. (2008) found that milk quality, in the form of bulk tank SCC, improved when premium and penalty programs were in place, indicating that dairy producers are highly responsive to incentives. Similar premium and penalty programs might be effective at improving dairy beef quality. Additionally, nonfinancial factors affecting decision 
making by dairy farmers in this area should be further investigated. A recent review by Edwards-Jones (2006) discusses financial and nonfinancial components that influence decision-making processes by farmers and highlights the need for further research that explores economic and psychological elements that affect decision making regarding animal health and welfare.

Ahola (2010) suggests that dairy cows are an important contributor to the nation's beef supply, and that an opportunity currently exists for the dairy industry to take advantage of the increasing value of market cull cows, ultimately increasing their income from the sale of these animals. To accomplish this, however, producers must provide better quality carcasses with fewer defects that result in fewer discounts. As an increasing percentage of each dairy cow carcass is being fabricated into whole muscle and higher priced cuts, injection site blemishes have historically prevented many dairy farmers from benefiting from this improved price (Ahola, 2010; Ahola et al., 2011). Dairy cows received $\$ 2.92$ (per 45.5 $\mathrm{kg}$ of BW) less at auction than beef cows, $\$ 12.87$ less than dairy bulls, and $\$ 14.07$ less than beef bulls (Ahola et al., 2011), which could be attributed to the fact that dairy cows are selected more often for carcass examination than the other classes of cattle. The main reasons a carcass will be selected for examination are signs that the entire carcass, or a part of it, is not fit for human consumption (USDA-FSIS, 2013), and may include evidence of disease, injection-site lesions, or surgical scars. Carcasses that get selected are examined more closely, with any suspect parts being cut from the carcass, resulting in loss of product. By following BQA guidelines and providing a better quality product at auction, dairy producers could see market cull cows accounting for much more than $4.6 \%$ of their annual income. In order for this to become a reality, however, dairy producers need to embrace the fact that they are producing not only milk and associated products, but beef as well.

As with any business, open communication between dairy ownership and management is an important step to a successful operation. Organizations in general tend to become more complex and add layers of management as production goals increase. These additional layers of management increase the disconnection between those at higher and lower levels of management and threaten organizational success (Glenn and Malott, 2004). To investigate the idea that ownership involvement with day-to-day operations decreases as operations get larger, we included questions in the current study to assess differences between owner and herdsman responses and determine if there is a lack of communication between those designing protocols and those actually working in the pens with the animals. Table 2 shows responses for owners and herdsmen to a series of questions pertaining to animal and worker injuries, BQA procedures, and record keeping. Of all of the questions asked, only one significant difference was found between the 2 groups. Interestingly enough, it concerned human workers, not dairy cows. All of the owners stated that no human workers had been injured while working with animals in the handling facility, whereas $33 \%$ of the herdsmen confirmed that injuries had actually occurred in the handling area. Although the severity of the injuries was not explored in this survey, it could explain the difference in perception of worker injuries. It seems likely that in the case of an injury not requiring medical attention, only the worker's immediate supervisor, the herdsman, would be aware of the injury. Injuries not requiring paperwork may not make it to the owner's attention, as minor injuries such as scrapes and bruises are commonplace when working in agriculture.

Caution must be taken when interpreting the lack of significance between differences in owner and herdsman responses because of the small number of dairies participating in the survey. Although not significantly different, a sizable difference was detected in the percentage of owners and herdsmen who stated that standard operating procedures for BQA existed, with a $29 \%$ difference between responses of the 2 groups. This difference could become more apparent when examined on a larger sample size that includes a greater spectrum of dairy operations in terms of priority given to handling systems and beef quality assurance. Results from this study provide valuable information for the design

Table 2. Owner and herdsman responses to questions, illustrating potential differences between owner perception and day-to-day operations on Colorado dairy farms

\begin{tabular}{|c|c|c|c|c|c|c|c|}
\hline \multirow[b]{2}{*}{ Question } & \multicolumn{3}{|c|}{ Owner } & \multicolumn{3}{|c|}{ Herdsman } & \multirow[b]{2}{*}{$P$-value } \\
\hline & Yes & No & Total no. & Yes & No & Total no. & \\
\hline Have any workers been injured in the last $12 \mathrm{mo} ?$ & 0 & 15 & 15 & 5 & 10 & 15 & $0.04^{\mathrm{a}}$ \\
\hline Have any animals been injured in the last 12 mo? & 6 & 9 & 15 & 7 & 8 & 15 & 1.000 \\
\hline Do you have standard operating procedures for $\mathrm{BQA}^{1}$ ? & 13 & 1 & 14 & 9 & 5 & 14 & 0.16 \\
\hline Are records kept on withdrawal times for all drugs administered? & 14 & 0 & 14 & 12 & 2 & 14 & 0.48 \\
\hline
\end{tabular}

${ }^{\mathrm{a}} \mathrm{A}$ difference was found between owner and herdsman responses $(P<0.05)$.

${ }^{1}$ Beef Quality Assurance procedures. 
of follow-up studies and a baseline of DBQA practices to measure changes resulting from future educational interventions or policy modification. Because of the small sample size of this pilot study, the researchers feel that larger scale studies should be conducted to determine if these DBQA practices are consistent within the Colorado dairy industry as well as other regions of the country.

\section{CONCLUSIONS}

The Colorado dairy industry is complex, with each operation being unique in terms of management practices, housing and facilities, production type, and areas of strength. Despite these differences, many similarities were apparent in the survey's results. Most of the owners and herdsmen agreed that the preferred location for i.m. and s.c. injections was in the neck area, but only a very small number confirmed that $100 \%$ of injections were given in that area. Dairy owners and herdsmen disagreed in whether or not workers were injured, which might suggest disconnection between upper and lower management. A handling facility that allows for the safe and efficient administration of all medications in the neck of dairy cows might prove beneficial, even if dairy producers consistently ranked administering injections according to BQA standards as last in priority when designing a new handling facility. In order for DBQA protocols to become commonplace on a dairy operation, the dairy industry must undergo a paradigm shift. Producers would benefit from embracing the idea that dairy animals produce not only milk and dairy products, but also beef products. Dairy producers must accept that they play a key role in ensuring that dairy beef is safe and high quality. Similar to their commitment to producing safe and nutritious milk for their customers, they must also be committed to producing the best quality beef. By making DBQA a priority, the dairy industry would not only benefit from increased profits from the sale of cull cows, but would also improve relationships and increase the confidence of processors, packers, and consumers.

\section{REFERENCES}

Ahola, J. K. 2010. Are market dairy cows undervalued? Beef Issues Q. 2:1-5.

Ahola, J. K., H. A. Foster, D. L. VanOverbeke, K. S. Jensen, R. L. Wilson, J. B. Glaze Jr., T. E. Fife, C. W. Gray, S. A. Nash, R. R. Panting, and N. R. Rimbey. 2011. Survey of quality defects in market beef and dairy cows and bulls sold through livestock auction markets in the Western United State: I. Incidence rates. J. Anim. Sci. 89:1474-1483.

Bickert, W. G., B. Holmes, K. Janni, D. Kammel, R. Stowell, and J. Zulovich. 2000. Special handling and treatment facilities. Pages 65-74 in Dairy Freestall Housing and Equipment. 7th ed. Midwest Plan Service, Ames, IA.

BQA (Beef Quality Assurance). 2012. National Manual. BQA, Centennial, CO.
Buttars, N. K., A. J. Young, and D. Bailey. 2006. Adoption of security systems by dairy farms to address bioterrorist threats in the Intermountain United States. J. Dairy Sci. 89:1822-1829.

Edwards-Jones, G. 2006. Modelling farmer decision-making: Concepts, progress and challenges. Anim. Sci. 82:783-790. http://dx.doi. org/10.1017/ASC2006112.

Glaze, J. B., Jr., and M. Chahine. 2009. Assessment of management of basic beef quality assurance practices on Idaho dairies. J. Dairy Sci. 92:1265-1271.

Glenn, S. S., and M. E. Malott. 2004. Complexity and selection: Implications for organizational change. Behav. Social Sci. 13:89-106.

Lindahl, C., P. Lundgvist, G. R. Hagevoort, C. Lunner Kolstrup, D. I. Douphrate, and T. Grandin. 2013. Occupational health and safety aspects of animal handling in dairy production. J. Agromedicine 18:274-283.

NBQA (National Beef Quality Audit). 2011. Executive Summary: The 2011 National Beef Quality Audit. National Cattleman's Beef Association, Centennial, CO.

NCBA (National Cattlemen's Beef Association). 2007. Executive Summary: 2007 National Market Cow and Bull Beef Quality Audit. NCBA's Beef Quality Assurance Program, Centennial, CO.

Nightingale, C., K. Dhuyvetter, R. Mitchell, and Y. Schukken. 2008. Influence of variable milk quality premiums on observed milk quality. J. Dairy Sci. 91:1236-1244.

Payne, M., C. M. Bruhn, B. Reed, A. Scearce, and J. O'Donnell. 1999. On-farm quality assurance programs: A survey of producer and industry leader opinions. J. Dairy Sci. 82:2224-2230.

Roeber, D. L. 2003. Meat Quality and Dairy Veterinary Practice. Pages 167-184 in Proc. Minnesota Dairy Health Conf. College Vet. Med., Univ. Minnesota, St. Paul.

Roeber, D. L., R. C. Cannell, W. R. Wailes, K. E. Belk, J. A. Scanga, J. N. Sofos, G. L. Cowman, and G. C. Smith. 2002. Frequencies of injection-site lesions in muscles from rounds of dairy and beef cow carcasses. J. Dairy Sci. 85:532-536.

Roeber, D. L., P. D. Miles, C. D. Smith, K. E. Belk, T. G. Field, J. D. Tatum, J. A. Scanga, and G. C. Smith. 2001. National market cow and bull beef quality audit-1999: A survey of producer-related defects in market cows and bulls. J. Anim. Sci. 79:658-665.

Rogers, C. A., A. C. Fitzgerald, M. A. Carr, B. R. Covey, J. D. Thomas, and M. L. Looper. 2004. On-farm management decisions to improve beef quality of market dairy cows. J. Dairy Sci. 87:15581564 .

Tozer, P. R., G. A. Varga, W. R. Henning, and L. A. Holden. 2005. Do dairy producers use effective management practices to improve the value of market cows? Prof. Anim. Sci. 21:272-277.

USDA (US Department of Agriculture). 2013. Actual slaughter under federal inspection. Accessed May 4, 2013. http://www.usda.gov/ nass/PUBS/TODAYRPT/lstk0413.pdf.

USDA-AMS (US Department of Agriculture Agricultural Marketing Service). 2012. National Organic Program: Organic Standards. USDA-AMS, Washington, DC. Accessed Apr. 25, 2013. http:// www.ams.usda.gov/AMSv1.0/nop.

USDA-APHIS (US Department of Agriculture Animal and Plant Health Inspection Service). 2008. Dairy 2007 Part II: Changes in the U.S. Dairy Cattle Industry, 1991-2007. USDA-APHIS, Fort Collins, CO.

USDA-FSIS (US Department of Agriculture Food Safety and Inspection Service). 2013. Slaughter inspection 101. USDA-FSIS, Washington, DC. Accessed Sep. 25, 2013. http://www.fsis.usda. gov/wps/portal/fsis/topics/food-safety-education/get-answers/ food-safety-fact-sheets/production-and-inspection/slaughterinspection-101/slaughter-inspection-101.

von Keyserlingk, M. A. G., N. P. Martin, E. Kebreab, K. F. Knowlton, R. J. Grant, M. Stephenson, C. J. Sniffen, J. P. Harner III, A. D. Wright, and S. I. Smith. 2013. Invited review: Sustainability of the US dairy industry. J. Dairy Sci. 96:5405-5425.

Wagner-Storch, A. M., and R. W. Palmer. 2002. Case study: Animal handling procedures and facilities used by dairy producers to conduct health and reproductive examinations. Prof. Anim. Sci. $18: 332-336$. 